\title{
On the accuracy of integral representation of differential operators in Lagrangian blob mesh-less methods
}

\author{
C. Golia \& B. Buonomo \\ Department of Aerospace and Mechanical Engineering, \\ Second University of Naples, Italy
}

\begin{abstract}
We explore novel ideas to improve the accuracy of the integral approximation of differential operators (Gradient and Laplacian) in the simulation of thermal viscous problems with Lagrangian Blob mesh-less methods.

Basically we investigate and develop a novel convolution integral discretization of the differential operators by using 2D-Taylor series expansions and a Gaussian like kernel function defined on a compact support around the blob centre of a given particle.

This allows us to overtake:

- deficiency of cells in the compact domain due to irregular distribution of the particles around the given blob,

- deficiency of cells in the compact domain caused by the presence of a boundary cutting the support of a nearby blob.

The accuracy and order of approximation of such a discretization are determined in regular and randomly distorted grids of various sizes, and compared with the widely used PSE (Particle Strength Exchange) formulation.

Results obtained in the solution of thermal buoyant problems at realistic values of the Grashoff number demonstrate validity and benefits of the novel findings.
\end{abstract}

Keywords: integral definition of differential operators, lagrangian mesh-less methods, vortex/thermal blobs, thermal buoyant problems. 


\section{Introduction}

Mesh-less methods (both 2 and 3 D) are of growing interest in the simulation of viscous unsteady fluid-dynamic problems. In particular, we focus on the analysis of the particle based Lagrangian approach to the Helmholtz formulation of the complete Navier-Stokes equations. Such analysis is made using individual discrete particles, blobs, that, as computational elements, transport (with the velocity induced by the vorticity field) momentum/vorticity and energy.

In the regularized vortex blob method, the discretization of the equations is made by considering N-blobs problems where vorticity/energy is represented, for a general particle located in $(\underline{\mathrm{x}}, \mathrm{t})$, by convolution integrals with kernel radial functions, $W(r), r=|\underline{x}|$, satisfying given normalization properties.

The accuracy of the solution depends on the approximation of the differential operators of the equations. Usually diffusive Laplacian operators are discretized according to the Particle Strength Exchange (PSE) method proposed by Degond and Mas-Gallic [1] and Gradient operators (needed in the buoyant term and in the $3 \mathrm{D}$ vorticity equation stretching term) according to a similar method proposed by Eldredge et al [2]. Both such discretizations are defined by convolution integrals with kernel radial functions derived according to the specific kernel function, W(r), used.

The discretization of each term is substituted in the equations and integrated over the volume around each blob particle: this will result in a time integration of N-body problem.

The discretization of the differential operators is quite accurate for blobs regularly distributed in the field and away from boundaries/discontinuities. Usually, to avoid inaccuracy due to distorted particle field, a regrid is used, after a number of integration steps, to project the field on a regular mesh.

The interest of the authors lay in the modeling of Unsteady Free Convection Buoyant Flows (Helmholtz formulation) characterized by strong unsteady start up phenomena (usually mushroom rising domes) and by a timely growing convective field that requires vanishing asymptotic boundary conditions (pressure closure).

Since regrid of the actual blob field on a regular symmetric grid will result in a somewhat viscous step, the authors are willing to search for formulations of the differential operators that are accurate also for distorted blob fields.

\section{Taylor convolution formulation}

We consider a function $\mathrm{f}(\underline{\mathrm{x}})=\mathrm{f}(\mathrm{x}, \mathrm{y})$, on a $2 \mathrm{D}$ field, represented by blobs located on a non regular grid and we are looking to compute the values of Gradient and Laplacian of the field at a given blob located at $\underline{x}_{0}=\left(x_{0}, y_{0}\right)$ by a convolution integral strategy on a compact domain around the blob, by using a kernel function $\mathrm{W}\left(\underline{x}^{\mathrm{x}} \underline{\underline{x}}_{0}, \mathrm{~h}\right)$.

Following an idea developed by Liu and Lui [3], we consider a Taylor expansion of $\mathrm{f}(\mathrm{x}, \mathrm{y})$ around the given point $\left(\mathrm{x}_{0}, \mathrm{y}_{\mathrm{o}}\right)$ truncated at the $3^{\text {rd }}$ order: 


$$
\mathrm{f}(\mathrm{x}, \mathrm{y})=\mathrm{f}\left(\mathrm{x}_{\mathrm{o}}, \mathrm{y}_{\mathrm{o}}\right)+\left.\Delta \mathrm{x} \mathrm{f}_{\mathrm{x}}\right|_{\mathrm{o}}+\left.\Delta \mathrm{yf} \mathrm{f}_{\mathrm{y}}\right|_{0}+\left.\frac{1}{2} \Delta \mathrm{x}^{2} \mathrm{f}_{\mathrm{xx}}\right|_{0}+\left.\Delta \mathrm{x} \Delta \mathrm{yf} \mathrm{f}_{\mathrm{xy}}\right|_{0}+\left.\frac{1}{2} \Delta \mathrm{y}^{2} \mathrm{f}_{\mathrm{yy}}\right|_{\mathrm{o}}+\ldots
$$

where: $\Delta \mathrm{x}=\mathrm{x}-\mathrm{x}_{0}, \Delta \mathrm{y}=\mathrm{y}-\mathrm{y}_{\mathrm{o}}$.

We consider a kernel function $\mathrm{W}\left(\underline{\mathrm{x}}-\underline{x}_{0}, h\right)$ that is compact on a domain $\Omega$ of radius $\mathrm{k}$ times the grid size, $\mathrm{h}$, around $\underline{\mathrm{x}}_{0}$. The kernel vanish on the boundary of $\Omega$ and has unitary zero-order moment, whereas all other $\mathrm{n}$-th order moments are zero:

$$
\begin{aligned}
& \mathrm{M}_{0} \equiv \int_{\Omega} \mathrm{W}\left(\underline{\mathrm{x}}-\underline{\mathrm{x}}_{0}, \mathrm{~h}\right) \mathrm{d} \underline{\mathrm{x}}=1 \\
& \mathrm{M}_{\mathrm{n}} \equiv \int_{\Omega}\left(\underline{\mathrm{x}}^{-} \underline{\mathrm{x}}_{0}\right)^{\mathrm{n}} \mathrm{W}\left(\underline{\mathrm{x}}-\underline{\mathrm{x}}_{0}, \mathrm{~h}\right) \mathrm{d} \underline{\mathrm{x}}=0 \quad \forall \mathrm{n} \neq 0
\end{aligned}
$$

Moreover we assume that the first partial derivatives of the kernel, $\mathrm{W}_{\mathrm{x}}\left(\underline{\mathrm{x}}-\underline{\underline{x}}_{0}, h\right)$ and $\mathrm{W}_{\mathrm{y}}\left(\underline{x}_{-} \underline{\mathrm{x}}_{0}, h\right)$, vanish on the boundary and have unitary first-order moments whereas all others vanish:

$$
\begin{aligned}
& \mathrm{M}_{1} \equiv \int_{\Omega}\left(\underline{\mathrm{x}}-\underline{\mathrm{x}}_{0}\right) \mathrm{W}_{\mathrm{a}}\left(\underline{\mathrm{x}}-\underline{\mathrm{x}}_{0}, \mathrm{~h}\right) \mathrm{d} \underline{\mathrm{x}}=-1 \\
& \mathrm{M}_{\mathrm{n}} \equiv \int_{\Omega}\left(\underline{\mathrm{x}}-\underline{\mathrm{x}}_{0}\right)^{\mathrm{n}} \mathrm{W}_{\mathrm{a}}\left(\underline{\mathrm{x}}-\underline{\mathrm{x}}_{0}, \mathrm{~h}\right) \mathrm{d} \underline{\mathrm{x}}=0 \quad ; \forall \mathrm{n} \neq 1, \quad \mathrm{a}=(\mathrm{x}, \mathrm{y})
\end{aligned}
$$

Finally we assume also that all second partial derivatives of the kernel, $\mathrm{W}_{\mathrm{xx}}\left(\underline{\mathrm{x}}-\underline{\mathrm{x}}_{0}\right.$, $h), \mathrm{W}_{\mathrm{xy}}\left(\underline{x}_{-} \underline{\mathrm{x}}_{0}, h\right)$ and $\mathrm{W}_{\mathrm{yy}}\left(\underline{\mathrm{x}}_{-} \underline{\mathrm{x}}_{0}, \mathrm{~h}\right)$ vanish on the boundary and have unitary second-order moments whereas all others vanish:

$$
\begin{aligned}
& \mathrm{M}_{2} \equiv \int_{\Omega}\left(\underline{\mathrm{x}}-\underline{\mathrm{x}}_{0}\right)^{2} \mathrm{~W}_{\mathrm{ab}}\left(\underline{\mathrm{x}}-\underline{\mathrm{x}}_{0}, \mathrm{~h}\right) \mathrm{d} \underline{\mathrm{x}}=2 \\
& \mathrm{M}_{\mathrm{n}} \equiv \int_{\Omega}\left(\underline{\mathrm{x}}-\underline{\mathrm{x}}_{0}\right)^{\mathrm{n}} \mathrm{W}_{\mathrm{ab}}\left(\underline{\mathrm{x}}-\underline{\mathrm{x}}_{0}, \mathrm{~h}\right) \mathrm{d} \underline{\mathrm{x}}=0 \quad ; \forall \mathrm{n} \neq 2, \mathrm{ab}=(\mathrm{x}, \mathrm{y}) \otimes(\mathrm{x}, \mathrm{y})
\end{aligned}
$$

Since we are looking for a convolution representation of operators, we multiply eq. (1), truncated to 2 nd order, alternatively, by the two first partial derivatives of the kernel function $\mathrm{W}\left(\underline{x}^{\mathrm{x}} \underline{\underline{x}}_{0}, h\right)$ and integrate on the compact domain.

We obtain then a system of two linear equations:

$$
\left\{\begin{array}{l}
\left.\mathrm{f}_{\mathrm{x}}\right|_{0} \int_{\Omega} \Delta \mathrm{xW}_{\mathrm{x}} \mathrm{d} \Omega+\left.\mathrm{f}_{\mathrm{y}}\right|_{0} \int_{\Omega} \Delta \mathrm{y} \mathrm{W} \mathrm{d} \Omega \approx \int_{\Omega}\left[\mathrm{f}-\mathrm{f}_{\mathrm{o}}\right] \mathrm{W}_{\mathrm{x}} \mathrm{d} \Omega \\
\left.\mathrm{f}_{\mathrm{x}}\right|_{0} \int_{\Omega} \Delta \mathrm{x} \mathrm{W}_{\mathrm{y}} \mathrm{d} \Omega+\left.\mathrm{f}_{\mathrm{y}}\right|_{0} \int_{\Omega} \Delta \mathrm{y} \mathrm{W}_{\mathrm{y}} \mathrm{d} \Omega \approx \int_{\Omega}\left[\mathrm{f}-\mathrm{f}_{\mathrm{o}}\right] \mathrm{W}_{\mathrm{y}} \mathrm{d} \Omega
\end{array}\right.
$$

where: $f=f(\underline{x}), f_{o}=f\left(\underline{x}_{o}\right)$.

The solution of eq. (5) furnishes the representation of the components of the field gradient, $\mathrm{f}_{\mathrm{x}} \mathrm{f}_{\mathrm{y}}$, at $\underline{x}_{0}$.

Similarly by multiplying alternatively eq. (1) by the three second partial derivatives of the kernel function $\mathrm{W}\left(\underline{x}_{-} \underline{x}_{0}, h\right)$, by truncating the expansion to the $3^{\text {rd }}$ order and by integrating on the compact domain $\Omega$, we can obtain the representation of the second derivatives needed for the Laplacian $\left(f_{\mathrm{xx}}, \mathrm{f}_{\mathrm{yy}}\right)$ by solving the system of 3 linear equations: 


$$
\begin{aligned}
& \left|\mathrm{f}_{\mathrm{xx}}\right|_{\mathrm{o}} \frac{1}{2} \int_{\Omega} \Delta \mathrm{x}^{2} \mathrm{~W}_{\mathrm{xx}} \mathrm{d} \Omega+\left.\mathrm{f}_{\mathrm{xy}}\right|_{\mathrm{o}} \int_{\Omega} \Delta \mathrm{x} \Delta \mathrm{yW} \mathrm{W}_{\mathrm{xx}} \mathrm{d} \Omega+\left.\mathrm{f}_{\mathrm{yy}}\right|_{0} \frac{1}{2} \int_{\Omega} \Delta \mathrm{y}^{2} \mathrm{~W}_{\mathrm{xx}} \mathrm{d} \Omega \approx \int_{\Omega} \Delta \mathrm{fW}_{\mathrm{xx}} \mathrm{d} \Omega-\left.\mathrm{f}_{\mathrm{x}}\right|_{\mathrm{o}} \int_{\Omega} \Delta \mathrm{xW} \mathrm{W}_{\mathrm{xx}} \mathrm{d} \Omega-\left.\mathrm{f}_{\mathrm{y}}\right|_{0} \int_{\Omega} \Delta \mathrm{yW} \mathrm{w}_{\mathrm{xx}} \mathrm{d} \Omega \\
& \left\{\left.\mathrm{f}_{\mathrm{xx}}\right|_{\mathrm{o}} \frac{1}{2} \int_{\Omega} \Delta \mathrm{x}^{2} \mathrm{~W}_{\mathrm{xy}} \mathrm{d} \Omega+\left.\mathrm{f}_{\mathrm{xy}}\right|_{\mathrm{o}} \int_{\Omega} \Delta \mathrm{x} \Delta \mathrm{yW} \mathrm{W}_{\mathrm{xy}} \mathrm{d} \Omega+\left.\mathrm{f}_{\mathrm{yy}}\right|_{\mathrm{o}} \frac{1}{2} \int_{\Omega} \Delta \mathrm{y}^{2} \mathrm{~W}_{\mathrm{xy}} \mathrm{d} \Omega \approx \int_{\Omega} \Delta \mathrm{fW}_{\mathrm{xy}} \mathrm{d} \Omega-\left.\mathrm{f}_{\mathrm{x}}\right|_{\mathrm{o}} \int_{\Omega} \Delta \mathrm{xW} \mathrm{W}_{\mathrm{xy}} \mathrm{d} \Omega-\left.\mathrm{f}_{\mathrm{y}}\right|_{\mathrm{o}} \int_{\Omega} \Delta \mathrm{yW} \mathrm{W}_{\mathrm{xy}} \mathrm{d} \Omega\right. \\
& \left|\mathrm{f}_{\mathrm{xx}}\right|_{\mathrm{o}} \frac{1}{2} \int_{\Omega} \Delta \mathrm{x}^{2} \mathrm{~W}_{\mathrm{yy}} \mathrm{d} \Omega+\left.\mathrm{f}_{\mathrm{xy}}\right|_{0} \int_{\Omega} \Delta \mathrm{x} \Delta \mathrm{yW}_{\mathrm{yy}} \mathrm{d} \Omega+\left.\mathrm{f}_{\mathrm{yy}}\right|_{\mathrm{o}} \frac{1}{2} \int_{\Omega} \Delta \mathrm{y}^{2} \mathrm{~W}_{\mathrm{yy}} \mathrm{d} \Omega \approx \int_{\Omega} \Delta \mathrm{fW}_{\mathrm{yy}} \mathrm{d} \Omega-\left.\mathrm{f}_{\mathrm{x}}\right|_{\mathrm{o}} \int_{\Omega} \Delta \mathrm{xW}_{\mathrm{yy}} \mathrm{d} \Omega-\left.\mathrm{f}_{\mathrm{y}}\right|_{\mathrm{o}} \int_{\Omega} \Delta \mathrm{yW} \mathrm{W}_{\mathrm{yy}} \mathrm{d} \Omega
\end{aligned}
$$

It is worthy to note that in case of symmetrical grid, many of the convolution integrals will vanish, and a much simple representations will result:

for the Gradient:

$$
\left.\mathrm{f}_{\mathrm{x}}\right|_{\mathrm{o}} \approx \frac{\int_{\Omega} \Delta \mathrm{fW}_{\mathrm{x}} \mathrm{d} \Omega}{\int_{\Omega} \Delta \mathrm{xW}_{\mathrm{x}} \mathrm{d} \Omega} \quad ;\left.\quad \mathrm{f}_{\mathrm{y}}\right|_{\mathrm{o}} \approx \frac{\int_{\Omega} \Delta \mathrm{fW}_{\mathrm{y}} \mathrm{d} \Omega}{\int_{\mathrm{v}} \Delta \mathrm{y} \mathrm{W}_{\mathrm{y}} \mathrm{d} \Omega}
$$

for the Laplacian:

$$
\begin{array}{r}
\left.\mathrm{f}_{\mathrm{xx}}\right|_{0} \approx \frac{1}{2} \frac{\int_{\Omega} \Delta \mathrm{f} \mathrm{W}_{\mathrm{xx}} \mathrm{d} \Omega * \int_{\Omega} \Delta \mathrm{y}^{2} \mathrm{~W}_{\mathrm{yy}} \mathrm{d} \Omega-\int_{\Omega} \Delta \mathrm{f}_{\mathrm{yy}} \mathrm{d} \Omega * \int_{\Omega} \Delta \mathrm{y}^{2} \mathrm{~W}_{\mathrm{xx}} \mathrm{d} \Omega}{\int_{\Omega} \Delta \mathrm{x}^{2} \mathrm{~W}_{\mathrm{xx}} \mathrm{d} \Omega * \int_{\Omega} \Delta \mathrm{y}^{2} \mathrm{~W}_{\mathrm{yy}} \mathrm{d} \Omega-\int_{\Omega} \Delta \mathrm{x}^{2} \mathrm{~W}_{\mathrm{yy}} \mathrm{d} \Omega * \int_{\Omega} \Delta \mathrm{y}^{2} \mathrm{~W}_{\mathrm{xx}} \mathrm{d} \Omega} \\
\left.\mathrm{f}_{\mathrm{yy}}\right|_{0} \approx \frac{1}{2} \frac{\int_{\Omega} \Delta \mathrm{x}^{2} \mathrm{~W}_{\mathrm{xx}} \mathrm{d} \Omega * \int_{\Omega} \Delta \mathrm{f} \mathrm{W}_{\mathrm{yy}} \mathrm{d} \Omega-\int_{\Omega} \Delta \mathrm{y}^{2} \mathrm{~W}_{\mathrm{yy}} \mathrm{d} \Omega * \int_{\Omega} \Delta \mathrm{f} \mathrm{W}_{\mathrm{xx}} \mathrm{d} \Omega}{\int_{\Omega} \Delta \mathrm{W}_{\mathrm{xx}} \mathrm{d} \Omega * \int_{\Omega} \Delta \mathrm{y}^{2} \mathrm{~W}_{\mathrm{yy}} \mathrm{d} \Omega-\int_{\Omega} \Delta \mathrm{x}^{2} \mathrm{~W}_{\mathrm{yy}} \mathrm{d} \Omega * \int_{\Omega} \Delta \mathrm{y}^{2} \mathrm{~W}_{\mathrm{xx}} \mathrm{d} \Omega}
\end{array}
$$

In the following we shall denote the representations given by eqs. (5) and (6) as "TCFC" and the representations given by eqs. (7) and (8) as "TCFP".

\section{Kernel function}

The kernel used is a modified (2D) Gaussian:

$$
\mathrm{W}(\mathrm{x}, \mathrm{y}, \mathrm{h})=\frac{4}{\pi} \frac{1}{\mathrm{~h}^{2}} \exp \left(-\frac{4\left(\mathrm{x}^{2}+\mathrm{y}^{2}\right)}{\mathrm{h}^{2}}\right)
$$

It can be shown that such kernel, with its derivatives, satisfy the Moment closures as per eqs. (2)-(4). The kernel of eq. (9) is compact on $\mathrm{r} / \mathrm{h} \leq 2$ with an approximation of 1.5E-7; its first derivatives are compact with an approximation of 1.5E-6, the second derivatives are compact with an approximation of 1.5E-5.

\section{Strategy of the performance analysis}

We assume a given known field on a regular or perturbed grid over a region and we proceed to compute the values of Gradient and Laplacian on a number of collocation points. Thereafter, we do compare the values computed with various methods with the exact ones and determine the statistics of the errors. 
Among others used, we report the analysis for the field:

$$
f(x, y)=\cos (2 \pi x) \sin (2 \pi y) /(2 \pi) \quad(x, y) \in[0,1] x[0,1]
$$

that represents the pressure field of a well known test case for benchmarking of Navier Stokes equations [4].

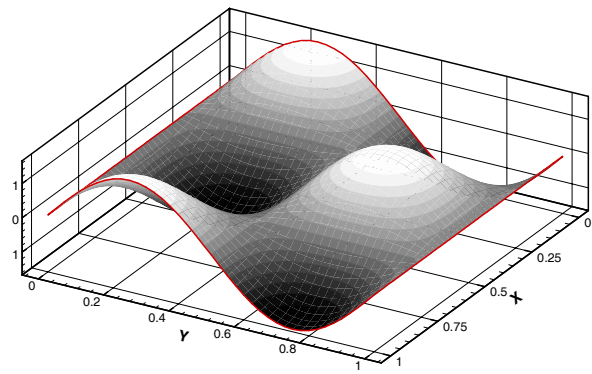

Figure 1.

Grid Comparison:

Igrid $=0$; NRandom $=0.5$
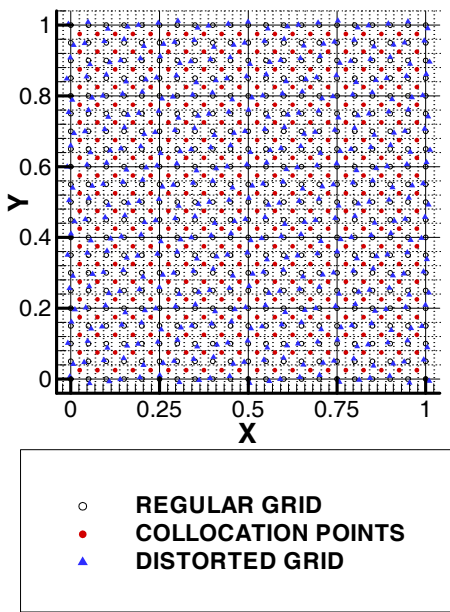

Figure 2.

We note that this field implies homogeneous Neumann conditions on the boundaries $\mathrm{x}=0$ and $\mathrm{x}=1$, and homogeneous Dirichlet conditions on the boundaries $\mathrm{y}=0$ and $\mathrm{y}=1$.

The reference grid is regular or perturbed according to the value of a random parameter $\mathrm{N}_{\text {random. }}$. For $\mathrm{N}_{\text {random }}=0$ the reference grid is symmetric. 
The grid of the collocation points, where we are computing the values of the Gradient and Laplacian, is located in the mid points of the original regular grid.

For various grid size and values of Nrandom, we will compare the performances of the methods: TCFC, TCFP, and PSE, in terms of:

- Field survey comparison maps,

- Map of the error in the field.

- Global RMS error,

For each formulation, the trend of the Global RMS error in terms of the grid size, $\mathrm{h}$, will give the real value of the order of approximation of the methods.

\subsection{Field survey comparison maps}

We show the Laplacian maps obtained with TCFC, TCFP, PSE, compared to the exact one for the case: $\mathrm{Nx} * \mathrm{Ny}=21 \cdot 21, \mathrm{~h}=0.05, \mathrm{~N}_{\text {random }}=0$ in Figure 3 .

We note that all the three methods catch the general behavior of the Laplacian field, but the PSE lacks for magnitude, and the TCFP locally suffers the Dirichlet boundary conditions on $\mathrm{y}=0$ and $\mathrm{y}=1$.
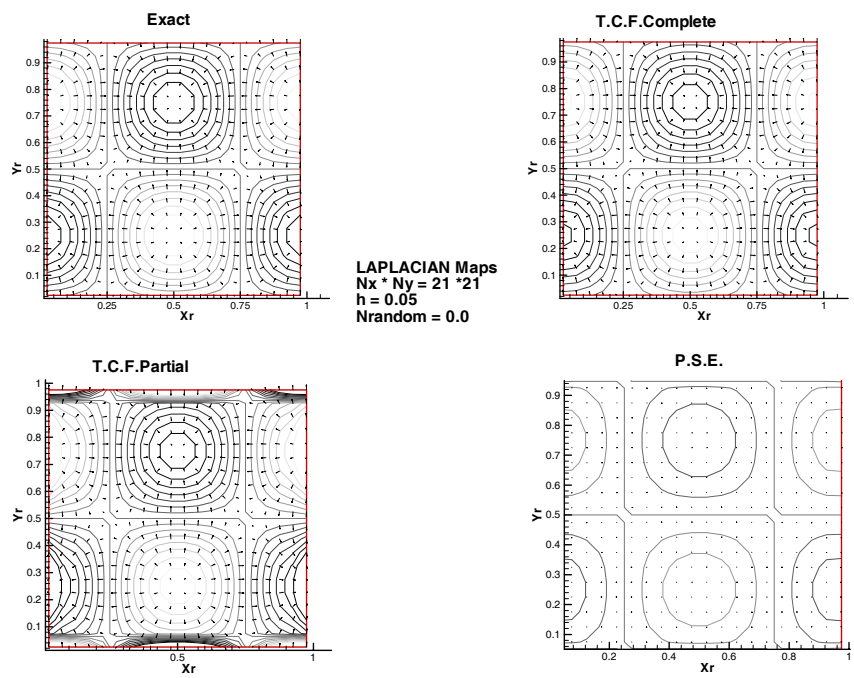

Figure 3.

The same case, obtained with a strongly distorted grid denoted by Nrandom $=0.5$, results in the maps in Figure 4.

It can be seen that TCFC is still able to give quantitative and qualitative satisfactory results, it suffers only the intersection zones with change of sign.

For the other two maps, TCFP is clearly failing and PSE is clearly unsatisfactory. 

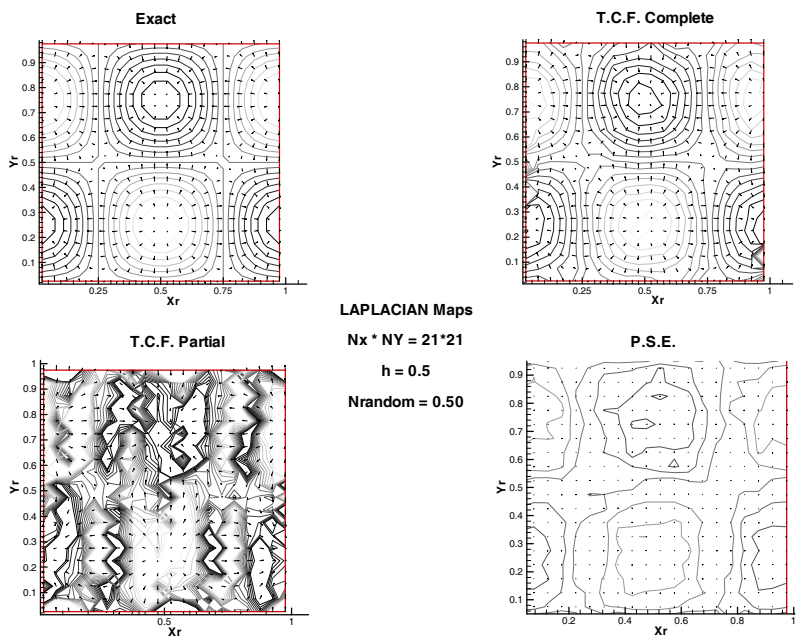

Figure 4.

\subsection{Field maps of the error}

For conciseness we report solely the error maps for TCFC and PSE corresponding to the case $\mathrm{Nx} * \mathrm{Ny}=41 * 11, \mathrm{~h}=0.025$, Nrandom $=0.25$ in Figure 5 .
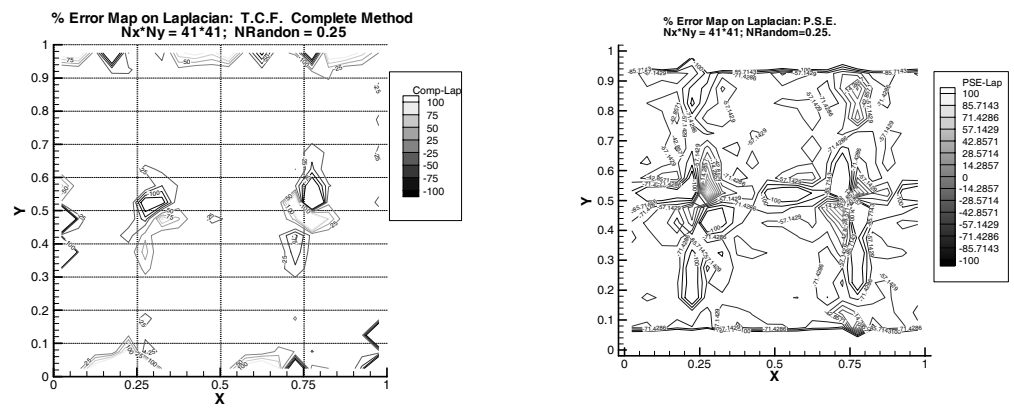

Figure 5.

These maps confirm what said in the previous paragraph. It is clear the much better accuracy of the TCFC.

\subsection{Error versus grid size}

We consider the error on the RMS norm of Gradient and Laplacian with varying grid size and distortion of the grid. Since we realize that such norms are affected by strong local errors nearby boundaries, we report the norm for the computations of the operators in collocation points within one grid size step away from the boundaries, that we call Inner grid.

We consider firstly the Gradient of the three methods. 


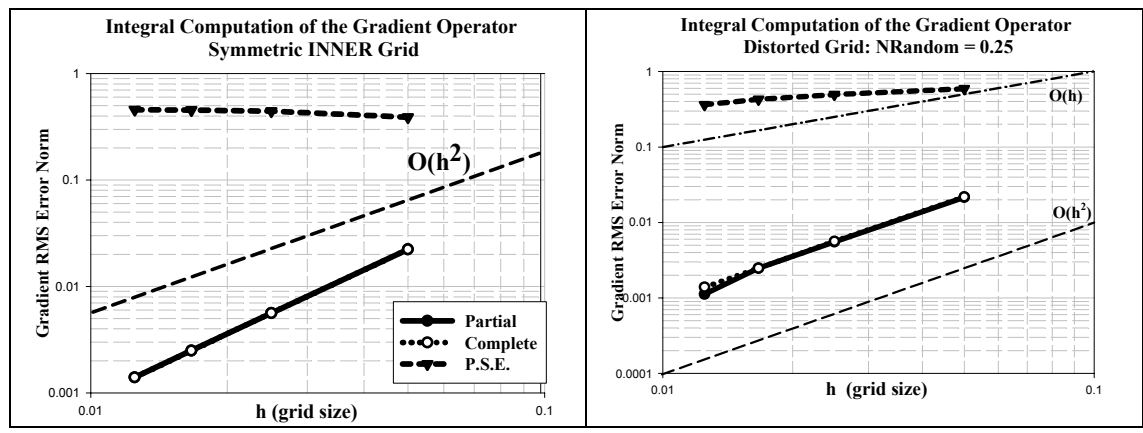

Figure 6.
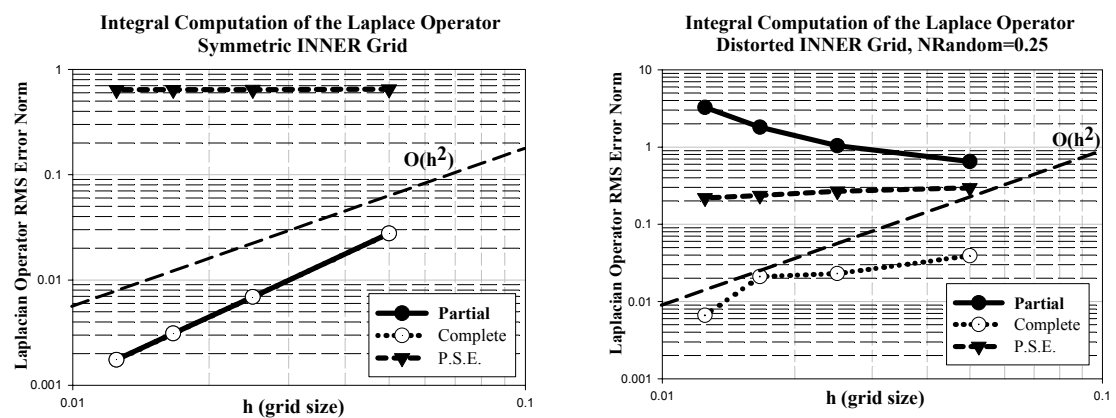

Figure 7.

The trends clearly show that TCFC and TCFP are almost equivalent, of 2nd order, and practically not sensible to grid distortion.

PSE is almost zero order. TCFC and clearly inadequate.

A comparison for the Laplacian shows that TCFC and TCFP are equivalent for symmetrical grid and $2^{\text {nd }}$ order. For distorted grid TCFC maintains roughly the $2^{\text {nd }}$ order accuracy, whereas TCFP and PSE are not well performing.

In conclusion, the TCFC perform much better of the other formulations both for Laplacian and for Gradients, and it is second order.

\section{Buoyant problem test}

We are going to compare the results of running the same 2D code with PSE and TCFC formulations of Gradients and Laplacians.

The code, detailed elsewhere in [5-7], is based upon Lagrangian particles (vortex and heat, independently treated) that, as computational elements, transport (with the velocity induced by the vorticity field) momentum/vorticity and energy. Vorticity is produced by the thermal gradient present in the flow field, in the context of validity of Boussinesq hypothesis. Heat is generated by the Thermal Boundary/Initial Conditions. Both vorticity and heat diffuse according to their transport coefficients and Laplacian terms. 
The formulation is based on a splitting technique of the convective and diffusive terms: it considers an hyperbolic equation for the trajectory of the blobs, and 2 parabolic equations for the diffusive processes, of thermal energy and vorticity, along the characteristics curves (trajectories).

The evaluation of the velocity from the vorticity field, needed to integrate the trajectories of the $\mathrm{N}$ blobs, is a classical N-Body problem that requires $\mathrm{O}\left(\mathrm{N}^{2}\right)$ operations. The code uses a Fast Multipole Method (FMM) that is a $\mathrm{O}(\mathrm{N})$ algorithm, capable of self organize in order to warrant an imposed error level on the calculation of the velocity field.

The resulting methodology has strong advantages, among the others: it is intrinsically unsteady, continuity is satisfied by definition, blobs move where they are needed (loci of strong gradients), asymptotic conditions are automatically satisfied, generates very robust codes (CFL condition removed). This will allow, with modern workstations and reasonable computing time, to simulate thermal free convection problems of engineering interest at realistic values of the Grashoff number.

The code is organized to perform, after a given number of computational steps, a regrid process, i.e. project the field on a regular grid. At time of the regrid the code automatically creates if it is the case, new blob particles to take into account the natural evolution of the flow.
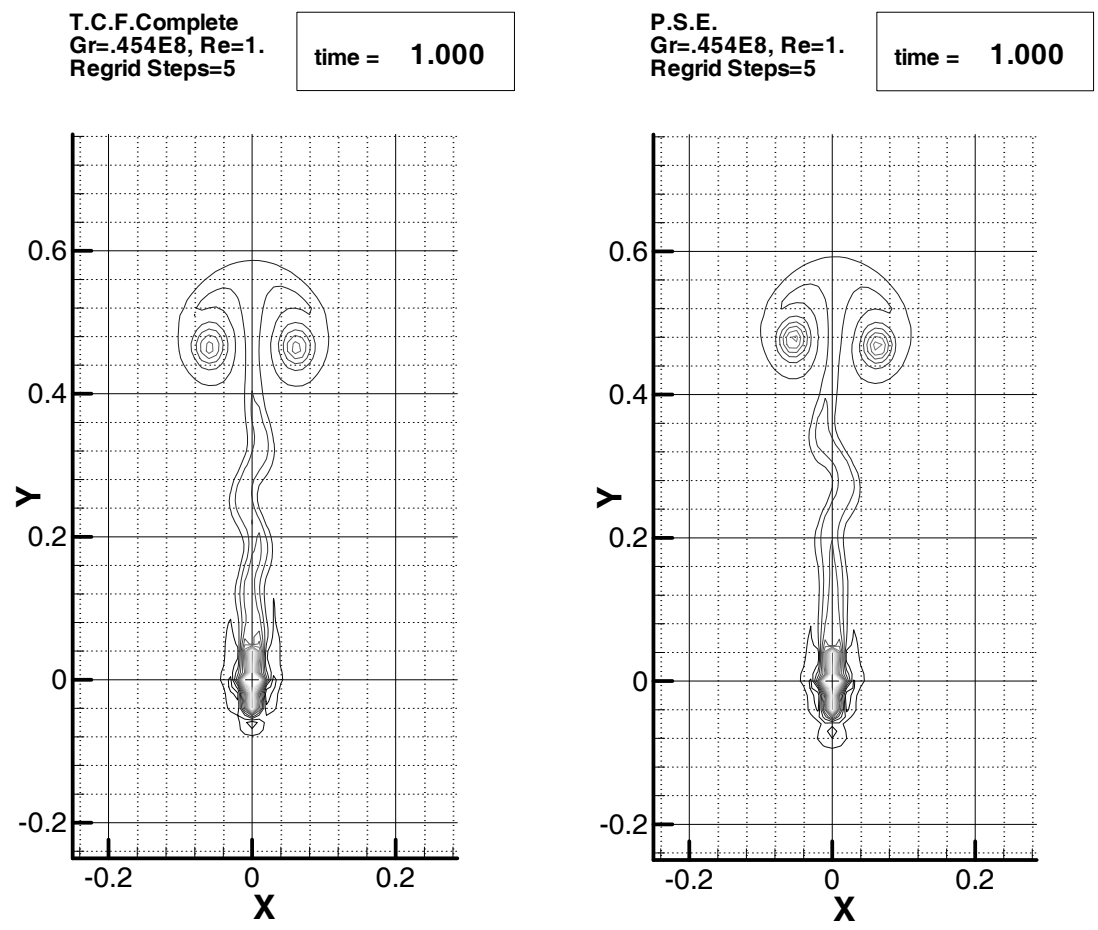

Figure 8. 
Since regrid steps result in a somewhat viscous step, object of this analysis is to compare the capacity of use a value of "Regrid Steps" as high as possible still preserving the main characteristics of the solutions.

First we use the same low Regrid Steps=5, for both cases, with a low resolution grid. The figure shows the isotherm maps.

We note that the solutions are very similar at initial stages, PSE slightly more varying at late times. This is caused, perhaps by the smoothing effect of the regrid processes. Then we run the same case, low resolution grid, with larger Regrid steps $=20$. The figure below shows isothermal maps and velocity plots. We can note that for the PSE, at late times the velocities grow with untidiness and this cause an abnormal break up of the mushroom rising cup and of its stem. Further analysis with a much finer grid reveals indeed shapes and mushroom rising cup very similar to the ones given by the TCFC.
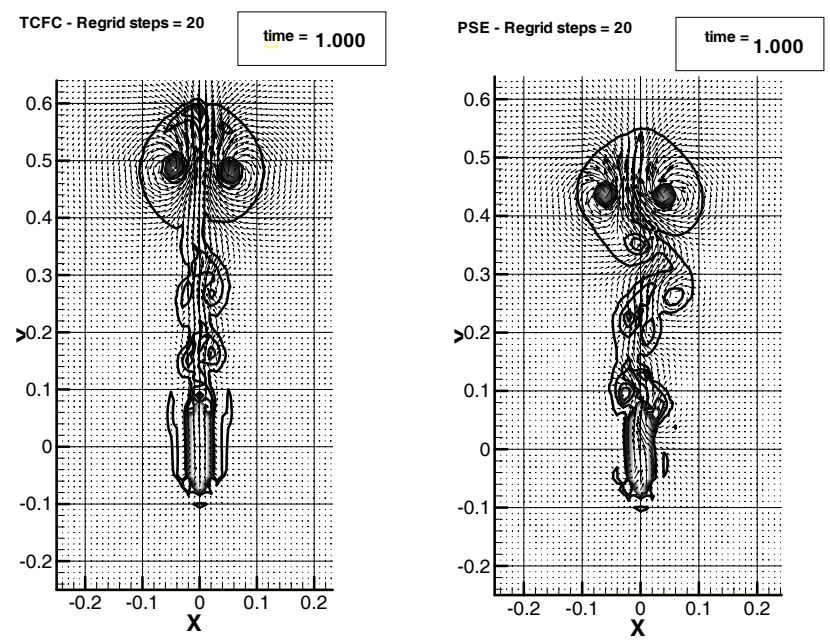

Figure 9.

\section{Conclusions}

Analysis and comparisons made in this paper confirm definitely the superiority of the TCFC for the representation of differential operators in blob mesh less methods. Preliminary tests with 3-D problems fully confirm such findings; details are reported in a companion paper [8].

\section{References}

[1] Degond, P. and Mas-Gallic, S., 1989, "The weighted Particle method for Convection-Diffusion Equations, part1: the case of an isotropic viscosity" Maths of Computation 53 (188), pp. 485-507

[2] Eldredge, J.D., Leonard, A. and Colonius, T., 2002, “A General Deterministic Treatment of Derivatives in Particle Methods", J. Comp. Phys. 180, pp. 686-709 
[3] Liu, G.R. and Liu, M.B., 2003, "Smoothed Particle Hydrodynamics", World Scientific Pub. Co.

[4] Ross Ethier, C. and Streinman, D.A., 1994, "Exact fully 3D Navier-Stokes solutions for benchmarking", Int. J. Num. Meth. in Fluids 19, pp. 369375.

[5] Golia, C., Buonomo, B., Manca, O., and Viviani, A., 2004, "A VortexThermal Blobs Method For Unsteady Buoyancy Driven Flows", ASMEIMECE. Anaheim, California.

[6] Golia, C., and Buonomo, B., 2005, "An Effective Blob Approach to Unsteady Thermal Buoyant Flows", CMEM 2005, Malta

[7] Golia, C., and Buonomo, B., 2005, "Numerical Simulation of Unsteady Natural Convection by Blobs Methods ", 60th ATI Congress, Rome, Italy

[8] Golia, C., Buonomo, B., and Viviani, A., 2007, "A corrected Vortex Blob Method for 3D Thermal Buoyan Flows”, ICTEA Amman, Jordan 